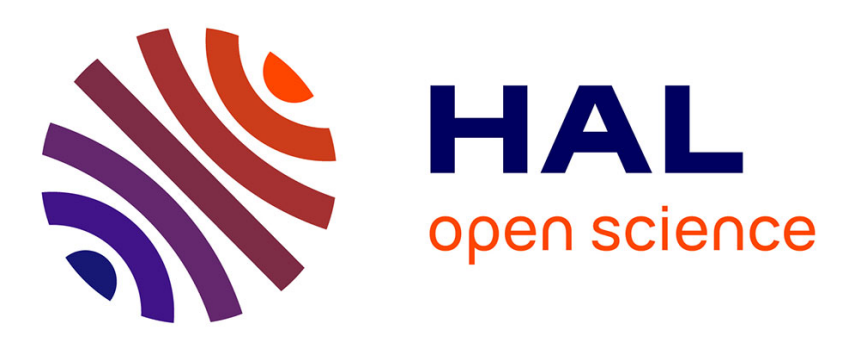

\title{
Modeling chemical flame structure and combustion dynamics in LES
}

Pierre Auzillon, Benoit Fiorina, Ronan Vicquelin, Nasser Darabiha, Olivier Gicquel, Denis Veynante

\section{- To cite this version:}

Pierre Auzillon, Benoit Fiorina, Ronan Vicquelin, Nasser Darabiha, Olivier Gicquel, et al.. Modeling chemical flame structure and combustion dynamics in LES. Proceeding of the combustion Institute, 2011, 33 (1), pp.1331-1338. 10.1016/j.proci.2010.05.045 . hal-00491238

\section{HAL Id: hal-00491238 \\ https://hal.science/hal-00491238}

Submitted on 10 Jun 2010

HAL is a multi-disciplinary open access archive for the deposit and dissemination of scientific research documents, whether they are published or not. The documents may come from teaching and research institutions in France or abroad, or from public or private research centers.
L'archive ouverte pluridisciplinaire HAL, est destinée au dépôt et à la diffusion de documents scientifiques de niveau recherche, publiés ou non, émanant des établissements d'enseignement et de recherche français ou étrangers, des laboratoires publics ou privés. 


\title{
Modeling chemical flame structure and combustion dynamics in LES
}

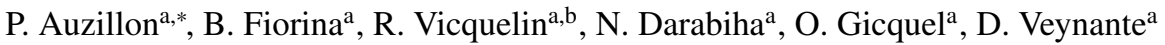 \\ ${ }^{a}$ EM2C - CNRS, Ecole Centrale Paris, Châtenay Malabry, France \\ ${ }^{b}$ GDF SUEZ, Pôle CHENE, Centre de Recherche et d'Innovation Gaz et Energies Nouvelles, 93211 Saint-Denis la Plaine, France
}

\begin{abstract}
In turbulent premixed combustion, the instantaneous flame thickness is typically thinner that the grid size usually retained in Large Eddy Simulations (LES), requiring adapted models. Two alternatives to couple chemical databases with LES balance equations, the Thickened Flame (TFLES) and the Filtered Tabulated Chemistry (F-TACLES) models, are investigated here and compared in terms of chemical flame structure and dynamics. To avoid the uncertainties related to the modeling of sub-grid scale turbulence / flame interactions, this comparison is conducted in situations where the flame front is not wrinkled at sub-grid scale levels. The thinner quantity requiring an accurate discretization on the numerical grid mesh is the reaction rate of the thickened or filtered progress variable. The thermal flame structure is found to be considerably thicker in TFLES than when using F-TACLES. The simulation of a 2D unsteady Bunsen burner flame shows that the thermal thickness spreading strongly affects the flame dynamics giving a decisive advantage to F-TACLES compared to TFLES.
\end{abstract}

Keywords:

Large Eddy Simulation, Turbulent premixed combustion, Tabulated chemistry

\section{Introduction}

Large Eddy Simulation is an attractive tool to predict flame dynamics and pollutant emissions in industrial combustion chambers [1]. The primary challenge is then to capture simultaneously the flame front propagation speed, the thermal expansion and the chemical species formation.

These phenomena are first driven by chemical kinetics and require therefore an accurate description of combustion chemistry. A widely used approach to describe fluid/chemistry interactions at a reduced computational cost is to tabulate chemical species mass fractions and/or reaction rates as functions of a reduced set of variables. A large variety of techniques has been developed to construct chemical databases. Among them, the ILDM (Intrinsic Low Dimensional Manifold) is based on a direct mathematical analysis of the dynamic behavior of the nonlinear chemical system response [2]. An alternative strategy is to map the chemical flame structure to a reduced phase subspace from elementary combustion configurations [3, 4]. For instance, in the Flame Prolongation of ILDM method

${ }^{*}$ Corresponding author : pierre.auzillon@em2c.ecp.fr
$[3,5]$, the chemical subspace of a turbulent premixed flame can be approximated by a collection of 1-D laminar flames. In such simple situations, all thermochemical quantities are related to a single progress variable related to the temperature or to a linear combination of chemical species. This tabulation chemistry method has been found very efficient and successful in laminar premixed and partially-premixed flames simulations $[3,6,7]$ and to compute turbulent premixed and partially-premixed flames in both RANS (Reynolds Averaged Navier-Stokes equations) $[8,9,10]$ and LES contexts $[11,12]$. As recently shown by Nguyen et al. [13], very complex flame structures such as triple flames can also be accurately recovered by tabulated chemistry techniques.

Unfortunately, an accurate description of the combustion chemistry is not sufficient to predict the correct flame front behavior in Large Eddy Simulations. The primary recurrent problem is that the flame thickness is typically thinner than the LES grid size. To overcome this issue, different strategies have been developed. The level-set or G-equation approach consists in tracking the inner layer by solving a propagation equation. Initially developed for RANS [14], the G-equation has been reformulated for LES [15, 16, 17]. However as level-set 
techniques provide information only on the thin reaction zone position and not on the filtered flame structure, the coupling with the flow equations remains challenging. In particular, an additional modeling effort is needed to estimate the temperature field and thermal expansion [18]. Moreover, the coupling between G-equation formalism and tabulated chemistry is a difficult task.

A common way to couple tabulated chemistry with turbulence in RANS [8, 9, 10] and in LES [19] is to presume the shape of the sub-grid-scale probability density function (PDF). Unfortunately, when extended to LES, this formulation does not guarantee a proper description of regimes where sub-grid-scale flame wrinkling vanishes [12]. These situations, observed when the sub-grid fluctuations are lower than the laminar flame speed, are encountered in practical LES of premixed combustion $[12,18,19]$. Additionally LES should tend toward DNS when the filter size becomes lower than the Kolmogorov scale.

A solution to propagate a flame front on a coarse grid is to artificially thicken the flame front (Thickened Flame model for LES) and to model subgrid scale wrinkling [20]. In premixed combustion, as this technique can be applied to the progress variable balance equation, coupling TFLES and tabulated chemistry is straightforward [21]. An alternative is to introduce a filter larger than the mesh size in order to resolve the chemical flame structure. Initially developed for infinitely thin flame front and for single-step chemistry [22, 23, 24], this strategy has recently been extended to tabulated chemistry in the Filtered Tabulated Chemistry for LES (FTACLES) model $[12,25]$. As the flame front is artificially spread in TFLES and F-TACLES approaches, flame structure and flow field may be affected.

The objective of this present work is to compare the effects of flame front thickening and filtering on both flame structure and dynamics. To avoid uncertainties related to the modeling of sub-grid-scale turbulence and flame interactions, the comparison is conducted in situations where the flame front is not wrinkled at the subgrid-scale level. TFLES and F-TACLES mathematical formalism are first briefly described in situations where flame wrinkling is fully resolved on the LES meshes (section 2). A criterion based on reaction zone thickness is proposed in section 3 to achieve quantitative comparison between the two competitive approaches. Finally, TFLES and F-TACLES models are applied to the simulation of an unsteady pulsed 2-D laminar Bunsen flame for various thickening factors and flame filter sizes, respectively (section 4). Results are compared to a filtered DNS solution serving as a reference solution.

\section{Coupling tabulated chemistry with LES}

\subsection{Problem formulation}

The FPI technique is retained here to determine lowdimensional trajectories covered by combustion chemistry $[3,5]$. For premixed combustion, one-dimensional freely propagating flames are first computed using detailed chemical schemes. Thermodynamical and chemical quantities are assumed to depend on a unique monotonic progress variable $c$, where $c=0$ corresponds to fresh gases and $c=1$ to fully burnt gases respectively. For instance, progress variable reaction rate is directly given by a look-up table: $\dot{\omega}_{c}=\dot{\omega}_{c}^{T a b}[c]$. The chemical database is then coupled to the flow field by adding a balance equation for the progress variable to the NavierStokes equations. For LES, under unity Lewis Number assumption, this equation is filtered leading to:

$$
\frac{\partial \bar{\rho} \widetilde{c}}{\partial t}+\nabla \cdot(\bar{\rho} \widetilde{\mathbf{u} c})=\nabla \cdot(\overline{\rho D \nabla c})-\nabla \cdot(\bar{\rho} \widetilde{\mathbf{u} c}-\bar{\rho} \widetilde{\mathbf{u} c})+\overline{\dot{\omega}}_{c}
$$

where $\rho$ is the density, $\mathbf{u}$ the velocity vector, $D$ is the diffusivity coefficient and $\dot{\omega}_{c}$ is the progress variable reaction rate. The overbar denotes the spatial filtering operation:

$$
\bar{\phi}(\mathbf{x})=\int_{\mathbb{R}^{3}} G\left(\mathbf{x}-\mathbf{x}^{\prime}\right) \phi\left(\mathbf{x}^{\prime}\right) d \mathbf{x}^{\prime}
$$

where $\phi$ represents reactive flow variables and velocity components and $G$ the filtering operator, here a Gaussian function:

$$
G(\mathbf{x})=\left(\frac{6}{\pi \Delta^{2}}\right)^{3 / 2} \exp \left(-\frac{6 \mathbf{x}^{2}}{\Delta^{2}}\right)
$$

where $\Delta$ is the filter size. The tilde operator denotes the density-weighted filtering defined by $\bar{\rho} \widetilde{\phi}=\overline{\rho \phi}$.

The sub-grid-scale transport term $-\nabla \cdot(\bar{\rho} \widetilde{\mathbf{u} c}-\bar{\rho} \widetilde{\mathbf{u} c})$, the filtered laminar diffusion term $\overline{\rho D \nabla c}$ and the filtered source term $\overline{\dot{\omega}}_{c}$, require closure models. When fully compressible solvers are considered, the closure of the energy balance equation must also be carefully addressed. For the sake of clarity, only the closure of the progress variable balance equation is discussed here. The full description of the F-TACLES model that include the closure of the filtered momentum and energy balance equations is given in [12].

The two possible approaches TFLES and F-TACLES to couple tabulated chemistry with flow field through the progress variable are now presented. 


\subsection{Introduction of tabulated chemistry in the Thick- ened Flame model}

A correct flame propagation on a coarse grid is ensured by artificially thickening the flame front [26]. Eq. (1) is then replaced by:

$$
\frac{\partial \bar{\rho} \widetilde{c}}{\partial t}+\nabla \cdot(\bar{\rho} \widetilde{\mathbf{u}} \bar{c})=\nabla \cdot(\bar{\rho} D F \nabla \widetilde{c})+\frac{\dot{\omega}_{c}^{T a b}[\widetilde{c}]}{F}
$$

where $F$ is the thickening factor. As both molecular diffusion and chemical source terms have been multiplied and divided by $F$ respectively, the flame front propagates at the same laminar flame speed $S_{l}^{0}$ than the original flame but is thickened by a factor of $F$ : $\widetilde{c}(\mathbf{x})=c(\mathbf{x} / F)$.

\subsection{Filtered Tabulated Chemistry model (F-TACLES)}

The F-TACLES approach solves the filtered progress variable balance equation (1) where unclosed terms are modeled from filtered one-dimensional laminar premixed flames. The flame structure in the direction $\mathbf{n}$ normal to the flame front is assumed identical to the structure of a planar 1-D freely-propagating premixed laminar flame, computed with a detailed chemical mechanism involving $N_{s}$ species and filtered using a Gaussian filter (Eq. 3) of size $\Delta$. The filtered source term for the filtered progress variable is then directly stored as a function of $\widetilde{c}$ and $\Delta$ in a filtered chemical database:

$$
\overline{\dot{\omega}}_{c}=\overline{\dot{\omega}}_{c}^{F T a b}[\widetilde{c}, \Delta]
$$

As discussed in [12], the filtered molecular diffusion term in Eq. (1) is modeled by:

$$
\nabla \cdot(\overline{\rho D \nabla c})=\nabla \cdot\left(\alpha_{c}^{F T a b}[\widetilde{c}, \Delta] \bar{\rho} D \nabla \bar{c}\right)
$$

where the correction factor $\alpha_{c}^{F T a b}[\widetilde{c}, \Delta]$ is defined as:

$$
\alpha_{c}^{F T a b}[\widetilde{c}, \Delta]=\overline{\rho D\left|\frac{\partial c^{*}}{\partial x^{*}}\right|}\left(\bar{\rho} D\left|\frac{\partial \widetilde{c^{*}}}{\partial x^{*}}\right|\right)^{-1}
$$

and estimated from filtered 1-D flame solutions and stored in a 2-D look-up table $[\widetilde{c}, \Delta]$. The superscript * corresponds to extraction from one-dimensional solution. Finally, assuming that the flame is not wrinkled by unresolved flow motions, the unresolved convection term can be estimated as [12]:

$$
\begin{aligned}
-\nabla \cdot(\bar{\rho} \widetilde{\mathbf{u}}-\bar{\rho} \widetilde{\mathbf{u} c}) & =-\rho_{0} S_{l}^{0}\left(\frac{\partial \overline{c^{*}}}{\partial x^{*}}-\frac{\partial \widetilde{c^{*}}}{\partial x^{*}}\right) \\
& =\Omega_{c}^{F T a b}[\widetilde{c}, \Delta]
\end{aligned}
$$

where $\Omega_{c}^{F T a b}[\widetilde{c}, \Delta]$ is also estimated from the filtered chemical database. The filtered progress variable balance equation for the model F-TACLES, neglecting subgrid-scale flame front wrinkling is therefore written:

$$
\begin{aligned}
\frac{\partial \bar{\rho} \widetilde{c}}{\partial t}+\nabla \cdot(\widetilde{\rho} \widetilde{\mathbf{u}}) & =\nabla \cdot\left(\alpha_{c}^{F T a b}[\widetilde{c}, \Delta] \bar{\rho} D \nabla \widetilde{c}\right) \\
+ & \Omega_{c}^{F T a b}[\widetilde{c}, \Delta]+\overline{\dot{\omega}}_{c}[\widetilde{c}, \Delta]
\end{aligned}
$$

The filtered energy balance equation is closed similarly when fully compressible flow are considered [12]. Situations where unresolved flame front wrinkling should be considered are also discussed in reference [12].

\section{Comparison of filtered and thickened flames}

TFLES and F-TACLES models described in Section 2 are implemented into the AVBP code [27]. The coupling of the chemical table and the compressible solver is ensured following Tabulated Thermo-chemistry for Compressible flows (TTC) formalism [28]. Planar onedimensional premixed methane/air flames are computed using GRI 3.0 mechanism [29] for an equivalence ratio equal to 1.05 to generate chemical databases. The ability of TFLES and F-TACLES to exactly reproduce planar laminar flame propagation speed has been already shown elsewhere $[1,12]$, but the present parametric study focuses on numerical grid requirements.

Two thicknesses are now introduced to characterize laminar premixed flames: the reaction zone thickness $\delta_{r}^{0}$, defined here as the full width at half maximum (FWHM) of the reaction rate $\dot{\omega}_{c}$, and the thermal thickness $\delta_{T}^{0}$, defined as the inverse of the maximum progress variable gradient $\left(\delta_{r}^{0}<\delta_{T}^{0}\right)$. Corresponding values for TFLES and F-TACLES flames are respectively $\left(\widehat{\delta}_{r}=\right.$ $\left.F \delta_{r}^{0}, \widehat{\delta}_{T}=F \delta_{T}^{0}\right)$ and $\left(\bar{\delta}_{r}, \bar{\delta}_{T}\right)$. Note that, when the filter size $\Delta$ is larger than the flame front thickness, as assumed in F-TACLES, $\bar{\delta}_{r} \approx \bar{\delta}_{T}$ while for TFLES $\widehat{\delta}_{r}<\widehat{\delta}_{T}$. Then, the lower progress variable scales to be resolved in the simulation on a numerical grid of mesh size, $\Delta_{x}$, are the reaction thicknesses $\widehat{\delta}_{r}$ and $\bar{\delta}_{r}$ in the TFLES and F-TACLES approaches respectively.

Figure 1 displays the predicted F-TACLES (respectively TFLES) flame front propagation speed ratio $S_{\Delta} / S_{l}^{0}$ (respectively $S_{F} / S_{l}^{0}$ ) as a function of the length scale ratio $\bar{\delta}_{r} / \Delta_{x}$ (respectively $\widehat{\delta}_{r} / \Delta_{x}$ ) for two values of the ratii $\bar{\delta}_{r} / \delta_{r}^{0}$ and $\widehat{\delta}_{r} / \delta_{r}^{0}$. The predicted filtered and thickened flame front propagation speeds, $S_{\Delta}$ and $S_{F}$, remain very close to the reference laminar flame speed $S_{l}^{0}$ as long as $\bar{\delta}_{r} / \Delta_{x}$ and $\widehat{\delta}_{r} / \Delta_{x}$ ratii remain larger than 3. Below this limit, a departure between computed and 
reference flame speeds is observed because of the underresolution of reaction rate profiles. Therefore, both minimal filtered and thickened reaction zone resolutions on a grid of cell size $\Delta_{x}$ are given by:

$$
\bar{\delta}_{r}^{\min }=\widehat{\delta}_{r}^{\min }=n \Delta_{x}
$$

where $n+1$ is the minimal number of grid points needed to resolve the flame front. The minimal thickening factor for TFLES is then: $F^{\min }=n \Delta_{x} / \delta_{r}^{0}$. For instance, present results show that flame simulations performed with AVBP using the third order TTGC numerical scheme [30] without artificial viscosity require $n=3$.

Thickening and filtering operators as defined in Section 2 are applied to the detailed chemistry solution to determine $\bar{\delta}_{r}(\Delta)$ and $\widehat{\delta}_{r}(F)$. The relation between $\mathrm{F}$ and $\Delta$ such as $\bar{\delta}_{r}(\Delta)=\widehat{\delta}_{r}(F)$ is then deduced and displayed in Fig. 2. In practice, this relation may be used to determine $\Delta^{\text {min }}$ from $\bar{\delta}_{r}\left(\Delta^{\text {min }}\right)=\widehat{\delta}_{r}\left(F^{\text {min }}\right)$. In Appendix A, an analytical relation between $F$ and $\Delta$ is derived modeling the progress variable reaction rate $\dot{\omega}_{c}$ by a Gaussian function and plotted in Fig. 2 (solid line).

Figure 3 shows the effect of the filtering and thickening operators on the flame structure when the resolved reaction zone thickness is preserved, i.e. when $\bar{\delta}_{r}=\widehat{\delta}_{r}$. Two reaction zone thicknesses corresponding to $F=2.5\left(\Delta=3 \delta_{r}^{0}\right)$ and $F=5.7\left(\Delta=7.9 \delta_{r}^{0}\right)$ are considered. Fig. 3(a) confirms that both filtered and thickened progress variable reaction rates have the same thickness but the thermal thickness, defined as the inverse of the progress variable gradient, is larger for TFLES than with F-TACLES. Figure 4 compares F-TACLES $\left(\bar{\delta}_{T}\right)$ and TFLES $\left(\widehat{\delta}_{T}\right)$ thermal flame thicknesses plotted as a function of the thickening factor $F=\widehat{\delta}_{r} / \delta_{r}^{0}$ when reaction layer thicknesses are identical $\left(\bar{\delta}_{r}=\widehat{\delta}_{r}\right)$. The thermal flame thickness is considerably more affected by TFLES than F-TACLES. As expected, $\bar{\delta}_{T} \approx \bar{\delta}_{r}$, while $\widehat{\delta}_{T} \approx 2.5 \widehat{\delta}_{r}$ (i.e. $\widehat{\delta}_{T} \approx 2.5 \bar{\delta}_{T}$ ). This behavior is also visible in Figs. 3(b) and 3(c), where temperature and velocity profiles are shown for both models. The response of a flame to a flow motion of size $r$ is primarily controlled by the ratio $r / \delta_{T}^{0}$. It has been shown in Ref. [20] that when the flame front is artificially broadened, then the flame response decreases. Therefore as $\widehat{\delta}_{T}>\bar{\delta}_{T}$ under similar mesh conditions, thickened flames will be always less affected by turbulence than filtered and original flames. In the next section, a 2-D unsteady laminar flame simulation is performed to verify these effects.

\section{Unsteady 2-D Bunsen flame simulation}

TFLES and F-TACLES formalisms are now tested against direct numerical simulations (DNS) to investigate the behavior of a bunsen laminar flame submitted to hydrodynamic excitation. Methane-air mixture at an equivalence ratio of 1.05 is issued from a $22 \mathrm{~mm}$ exit diameter nozzle burner. The inlet flow velocity is modulated harmonically according to $\bar{v}=0.97 \mathrm{~m} . \mathrm{s}^{-1}$, $v_{R M S}=0.19 \mathrm{~m} . \mathrm{s}^{-1}$ and $f=62.5 \mathrm{~Hz}$ where $\bar{v}, v_{R M S}$ and $f$ are the mean inflow velocity, the inflow velocity RMS and the excitation frequency. This configuration is a 2D simplification of the Schuller et al. [31] experimental set-up.

One-dimensional laminar methane-air flames are computed using the PREMIX software [32] and the GRI 3.0 chemical mechanism [29] to generate chemical look-up tables as a function of the progress variable defined from carbon monoxide and dioxide mass fractions as $c=\left(Y_{\mathrm{CO}}+Y_{\mathrm{CO}_{2}}\right) /\left(Y_{\mathrm{CO}}^{e q}+Y_{\mathrm{CO}_{2}}^{e q}\right)$ where eq superscript denotes equilibrium state.

The objective of the simulations is to evaluate the ability of F-TACLES and TFLES formalisms to reproduce unsteady flame behavior when flow motions are fully resolved (no sub-grid scale flame front wrinkling) but not the internal flame structure. A direct numerical simulation (DNS) is first performed on a 1.1 million grid elements to fully resolve the laminar flame structure. The mesh has been designed to ensure that at least 15 nodes and 6 nodes are present in the thermal and reaction zones, respectively. The smallest unsteady flow structure is resolved over approximately 20 nodes. From the DNS solution, a reference "LES" field is then obtained by filtering the progress variable field with the operator defined in Eq. (3).

LES have been performed with both F-TACLES and TFLES formalisms on two coarse grids of 0.6 and 0.3 millions of elements, respectively. On each grid, the reaction zone thicknesses of both models are set identical. The meshing criterion (Eq. 10) is respected (four grid points across $\bar{\delta}_{r}$ ). Model parameters are summarized in Table 1 . Results are analyzed after ten excitation cycles to ensure the independence of the solution from initial conditions.

Figures 5 and 6 show consecutive sequences of the pulsed Bunsen flame front position during an excitation cycle. F-TACLES (dashed-dotted lines) and TFLES (dotted lines) are compared to the reference DNS solution plotted with solid lines. For moderate values of filter size relative to the reaction thickness i.e. $\Delta=3 \delta_{r}^{0}$, a very good agreement is observed between F-TACLES and the DNS (Fig. 5) while the corresponding thickened 
flame solution obtained with $F=2.5$ slightly differs from the reference solution. The heat release rate integrated over the computational domain, displayed in Fig. 7 along an excitation cycle, is very well predicted by both approaches.

When the filter size increases up to $\Delta=7.9 \delta_{r}^{0}$, the flame dynamics remains fairly reproduced by $\mathrm{F}$ TACLES simulation while the TFLES solution obtained with $F=5.7$ fails to predict the flame patterns (Fig. 6). The heat release prediction is strongly impacted as shown in Fig. 8. F-TACLES predictions remain in good agreement even if a slight phase shift corresponding to a time lag of $0.1 / f$ is noticed. On the other hand, TFLES misses most of the unsteady behavior of the global reaction rate. As explained previously, for a given reaction layer thickness, the thermal thicknesses are differently affected in TFLES or F-TACLES approaches. As indicated in Table 1, the thickened thermal structure is 2.5 times larger than the reactive layer while the F-TACLES thermal layer remains of the same order than the filtered reaction zone.

It has been shown in Ref.[31] that a flame behaves as a low-pass filter. The thermal layer thickness broadening tends to decrease the filter cut-off frequency, increasing the flame response time. As discuss previously, the problem has three main length scales: $r$, the size of flow motions, $\bar{\delta}_{T}$ and $\widehat{\delta}_{T}$, the thermal resolved flame thicknesses when using F-TACLES and and TFLES respectively $\left(\widehat{\delta}_{T}>\bar{\delta}_{T}\right)$. When $r>>\widehat{\delta}_{T}>\bar{\delta}_{T}$, the flame wrinkling will be fully resolved and both models are expected to provide similar results. When $\widehat{\delta}_{T}>r>\bar{\delta}_{T}$. FTACLES is still expected to provide good results while TFLES will damp a part of the flame wrinkling. As discussed in section 3, for similar meshes leading to similar reaction thickness, the thermal flame thickness is larger with TFLES than with F TACLES. Therefore more flow structures will be damped by TFLES than F-TACLES. F-TACLES is then able to explicitly resolve a larger part of the flame turbulence interactions than TFLES.

\section{Conclusion}

The ability of TFLES and F-TACLES approaches to reproduce flame structure and dynamics has been investigated. Relevant comparison between both models are performed for identical filtered and thickened reaction layer thicknesses $\left(\widehat{\delta}_{r}=\bar{\delta}_{r}\right)$ that were found to be the thinnest zone to be resolved on a given numerical grid. The flame structure and consequently the flow field are more affected by the thickening operation (TFLES) than by the filtering procedure (F-TACLES). In fact, the thermal thickness of the resolved flame front is of the same order of the reaction zone thickness when using F-TACLES $\left(\bar{\delta}_{T} \approx \bar{\delta}_{r}\right)$ while it is larger, here by a factor of about 2.5, for TFLES. Simulations of a twodimensional Bunsen flame submitted to acoustic excitations show that the spreading of the thermal thickness strongly impacts the flame dynamics and might affect the prediction of combustion instabilities, a point to be investigated in the future. However, the F-TACLES formalism is expected to be more efficient than TFLES to describe flame dynamics and chemical flame structure in turbulent combustion regimes.

\section{Acknowledgments}

This work was supported by the ANR-07-CIS7008-04 Grant of the French Ministry of Research and accessed to the high performance computing resources of IDRIS and CINES under the allocation 2009i2009020164 made by GENCI (Grand Equipement National de Calcul Intensif). The authors also gratefully acknowledge Drs. T. Schuller and D. Durox for helpful discussions about their experiments.

\section{References}

[1] T. Poinsot, D. Veynante, Theoretical and Numerical Combustion, R. T. Edwards, Inc., 2005.

[2] U. Maas, S. B. Pope, in: Proc. Combust. Inst. 24, 1992, pp. $103-112$.

[3] O. Gicquel, N. Darabiha, D. Thévenin, in: Proc. Combust. Inst. 28, 2000, pp. 1901-1908.

[4] J. A. van Oijen, F. A. Lammers, L. P. H. de Goey, Combust. Flame 127 (3) (2001) 2124-2134

[5] G. Ribert, M. Champion, O. Gicquel, N. Darabiha, D. Veynante, Combust. Flame 141 (2005) 271-280.

[6] B. Fiorina, O. Gicquel, L. Vervisch, S. Carpentier, N. Darabiha, Combust. Flame 140 (3) (2005) 147-160.

[7] B. Fiorina, R. Baron, O. Gicquel, D. Thévenin, S. Carpentier, N. Darabiha, Combust. Theory and Model. 7 (2003) 449-470.

[8] B. Fiorina, O. Gicquel, L. Vervisch, S. Carpentier, N. Darabiha, Proc. Combust. Inst. 30 (2005) 867-874.

[9] L. Vervisch, R. Haugel, P. Domingo, M. Rullaud, J. of Turbulence 5 (4) (2004) 1-36.

[10] B. Fiorina, O. Gicquel, D. Veynante, Proc. Combust. Inst. 32 (2) (2009) 1687-1694.

[11] J. Galpin, A. Naudin, L. Vervisch, C. Angelberger, O. Colin, P. Domingo, Combust. Flame 155 (2008) 247-266.

[12] B. Fiorina, R. Vicquelin, P. Auzillon, N. Darabiha, O. Gicquel, D. Veynante, Combust. Flame 157 (2010) 465-475.

[13] P. Nguyen, L. Vervisch, V. Subramanian, P. Domingo, Combust. Flame 157 (1) (2010) 43-61.

[14] N. Peters, Turbulent Combust., Cambridge University Press, 2000.

[15] S. Menon, W. Jou, Combust. Sci. and Tech. 75 (1991) 53-72.

[16] V.-K. Chakravarthy, S. Menon, Flow, Turbulence and Combust. 65 (2000) 133-161.

[17] H. Pitsch, Combust. Flame 143 (4) (2005) 587-598.

[18] V. Moureau, B. Fiorina, H. Pitsch, Combust. Flame 156 (4) (2009) 801-812. 
[19] H. Pitsch, Ann. Rev. Fluid Mech. 38 (2006) 453-482.

[20] O. Colin, F. Ducros, D. Veynante, T. Poinsot, Physics of Fluids 12 (7) (2000) 1843-1863.

[21] G. Albouze, T. Poinsot, L. Gicquel, Comptes Rendus Mécanique 337 (6-7) (2009) 319-328.

[22] M. Boger, D. Veynante, H. Boughanem, A. Trouvé, Proc. Combust. Inst. 27 (1998) $917-925$.

[23] C. Duwig, Flow Turbulence and Combust. 79 (4) (2007) $433-$ 454.

[24] C. Duwig, Combust. Theory and Model. 13 (2) (2009) 251-268.

[25] R. Vicquelin, B. Fiorina, N. Darabiha, O. Gicquel, D. Veynante, Comptes Rendus Mécanique 337 (6-7) (2009) 329-339.

[26] T. D. Butler, P. J. O'Rourke, Proc. Combust. Inst. 16 (1977) 1503-1515.

[27] http://www.cerfacs.fr/cfd/avbp code.php.

[28] R. Vicquelin, B. Fiorina, S. Payet, N. Darabiha, O. Gicquel, Submitted to the Proc. Combust. Inst. 33.

[29] http://www.me.berkeley.edu/gri-mech/.

[30] O. Colin, M. Rudgyard, J. Comput. Phys. 162 (2) (2000) 338371.

[31] T. Schuller, S. Ducruix, D. Durox, S. Candel, Proc. Combust. Inst. 2929 (2002) 107-113.

[32] R. J. Kee, J. F. Grcar, M. D. Smooke, J. A. Miller, Tech. rep., Sandia National Laboratories (1992). 


\section{Appendix A. Analytical relation between the filter} size $\Delta$ and the thickening factor $F$

The laminar reaction rate is modeled as a Gaussian function:

$$
\dot{\omega}_{c}(x)=\rho_{0} S_{l} G\left(e_{r}, x\right) \quad \text { with } \quad e_{r}=\left(\frac{3}{2 \ln (2)}\right)^{1 / 2} \delta_{r}^{0}
$$

where $\delta_{r}^{0}$ is the full width at half maximum of the progress variable reaction rate. In the expression $e_{r}$ is chosen so that the integral of the progress variable source term and its full width at half maximum are respectively equal to those obtained by detailed chemistry 1-D calculations done with PREMIX code. $G(a, x)$ is the Gaussian function of size $a$ defined as :

$$
G(a, x)=\left(\frac{6}{\pi a^{2}}\right)^{1 / 2} \exp \left(-\frac{6 x^{2}}{a^{2}}\right)
$$

In TFLES approach, the thickened progress variable reaction rate reads:

$$
\widehat{\dot{\omega}}_{c}(x)=\frac{1}{F} \dot{\omega}_{c}\left(\frac{x}{F}\right)=\rho_{0} S_{l} G\left(F e_{r}, x\right)
$$

The F-TACLES source term is written, for a filter size $\Delta$ :

$$
\overline{\dot{\omega}}_{c}(x)=\rho_{0} S_{l} G\left(\left(\Delta^{2}+e_{r}^{2}\right)^{1 / 2}, x\right)
$$

remembering that filtering a Gaussian function of thickness $e_{r}$ with a Gaussian filter of size $\Delta$ corresponds to a Gaussian function of size $\left(e_{r}^{2}+\Delta^{2}\right)^{1 / 2}$. According to Eqs. (A.3) and (A.4), the condition $\bar{\delta}_{r}=\widehat{\delta}_{r}$ gives :

$\widehat{\dot{\omega}}_{c}(x)=\overline{\dot{\omega}}_{c}(x), F=\left(1+\frac{\Delta^{2}}{e_{r}^{2}}\right)^{1 / 2}=\left[1+\frac{2 \ln (2)}{3}\left(\frac{\Delta}{\delta_{r}^{0}}\right)^{2}\right]^{1 / 2}$ 


\begin{tabular}{|c||c|c|c|c|}
\hline Case & Model & $\delta_{r} / \delta_{r}^{0}$ & $\delta_{T} / \delta_{r}^{0}$ & cells \\
\hline \hline DNS & - & 1 & 2.6 & $1.1 \bar{M}$ \\
\hline \hline F 1 & F-TACLES $\Delta=3 \delta_{r}^{0}$ & 2.5 & 2.9 & $0.6 \bar{M}$ \\
\hline T 1 & TFLES $F=2.5$ & 2.5 & 6.5 & $0.6 \bar{M}$ \\
\hline \hline F 2 & F-TACLES $\Delta=7.9 \delta_{r}^{0}$ & 5.7 & 6.5 & $0.3 \bar{M}$ \\
\hline T 2 & TFLES $F=5.7$ & 5.7 & 14.8 & $0.3 \bar{M}$ \\
\hline
\end{tabular}

Table 1: Computational parameters of the 2-D unsteady Bunsen flame 

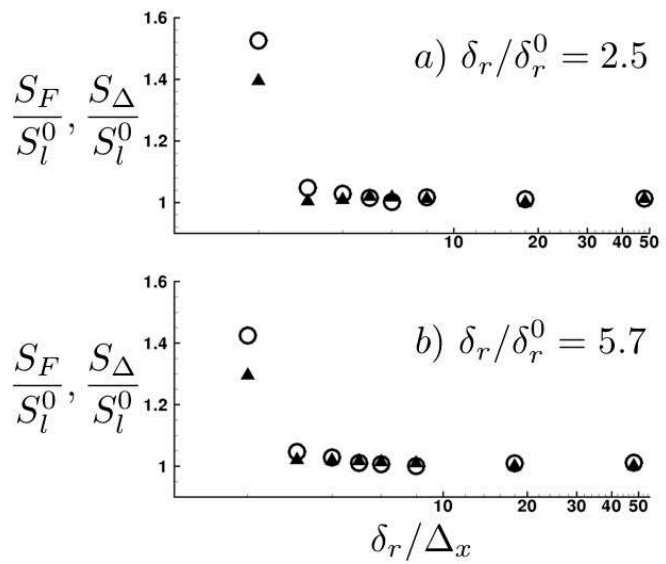

Figure 1: Predicted flame speed ratii as a function of $\bar{\delta}_{r} / \Delta_{x}$ for $\bar{\delta}_{r}=$ $\widehat{\delta}_{r}=2.5 \delta_{r}^{0}$ (top) and $\bar{\delta}_{r}=\widehat{\delta}_{r}=5.7 \delta_{r}^{0}$ (bottom). $\bigcirc$ : F-TACLES $S_{\Delta} / S_{l}^{0}$, $\boldsymbol{\Delta}$ : TFLES $S_{F} / S_{l}^{0}$.

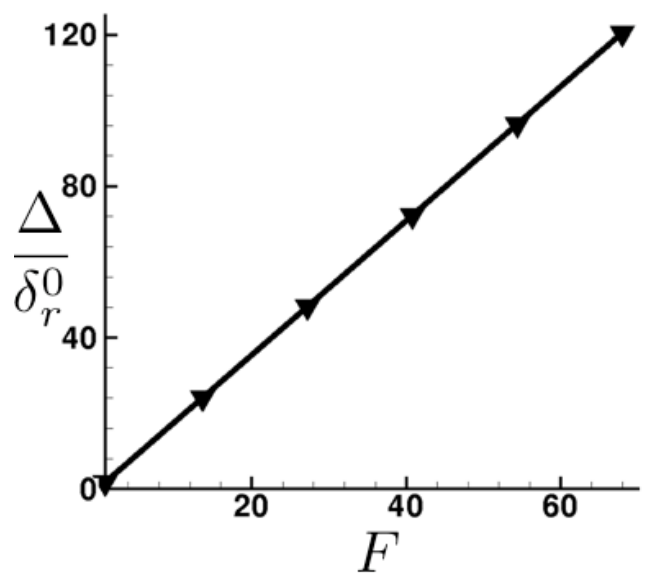

Figure 2: Relation between $\Delta / \delta_{r}^{0}$ and $F$ when $\bar{\delta}_{r}=\widehat{\delta}_{r}$. Symbols: Computations. Solid line: Analytical model (Appendix A).

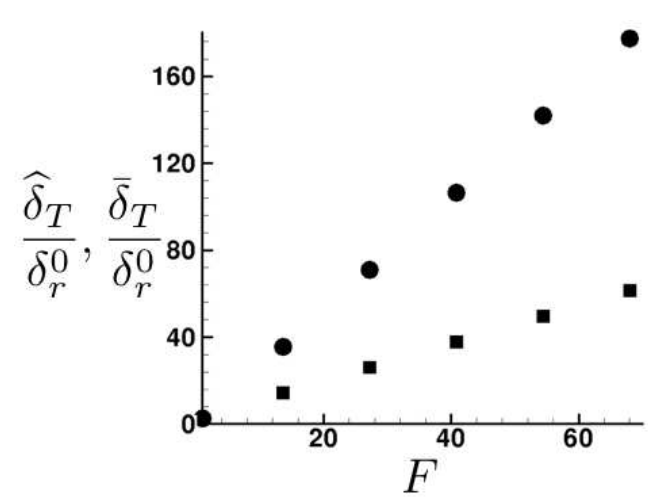

Figure 4: Evolution of the resolved thermal thickness as a function of the thickening factor $F, \mathbf{\square}: \bar{\delta}_{T}, \bullet: \widehat{\delta}_{T}$

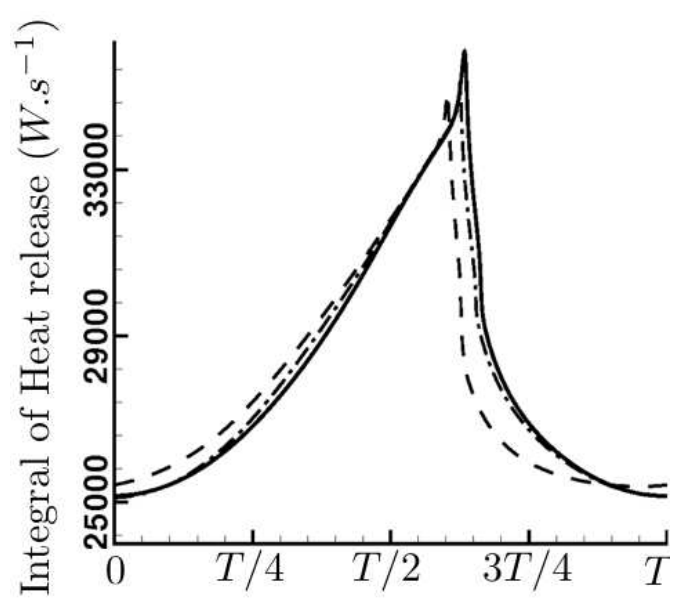

Figure 7: Heat release rate integrated over the computational domain as a function of time during a cycle. $\bar{\delta}_{r}=\widehat{\delta}_{r}=2.5 \delta_{r}^{0} ;-\mathrm{DNS} ; \cdot-\cdot$ F-TACLES; - - - TFLES 

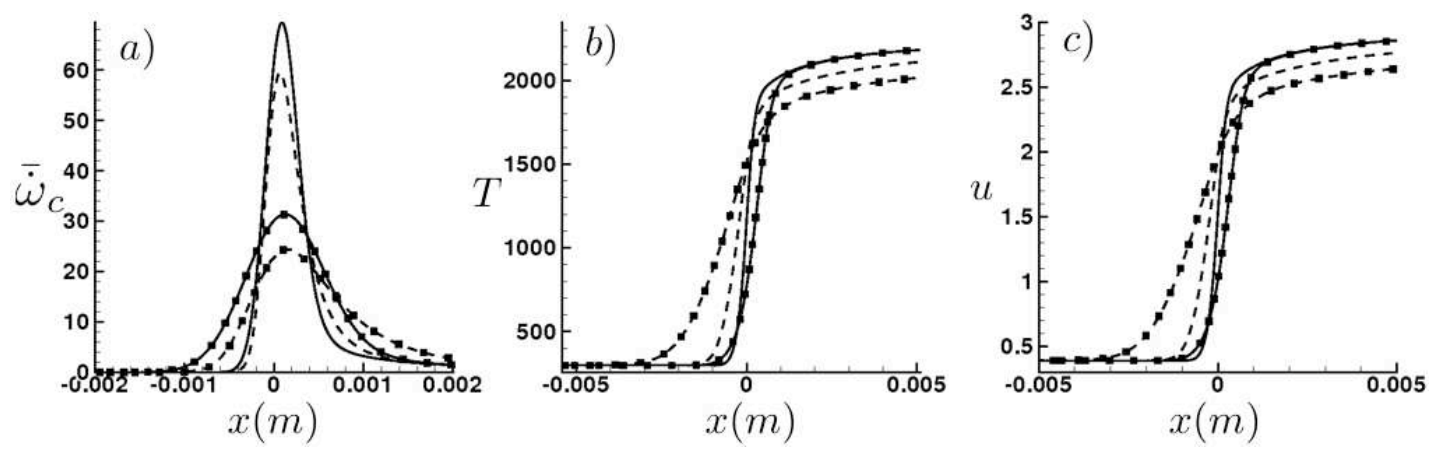

Figure 3: Effect of filtering and thickening operators on the flame structure. Solid lines: F-TACLES. Dashed lines: TFLES. Without symbol: $\bar{\delta}_{r}=\widehat{\delta}_{r}=2.5 \delta_{r}^{0}$. Symbols: $\bar{\delta}_{r}=\widehat{\delta}_{r}=5.7 \delta_{r}^{0}$. a) Progress variable source term, b) Temperature, c) Velocity
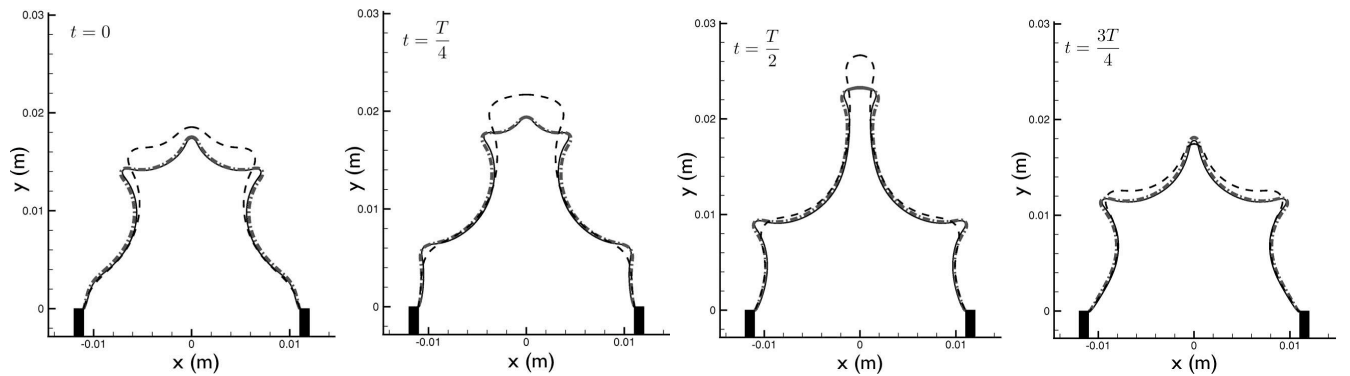

Figure 5: Iso-line $\widetilde{c}=0.5$ during a cycle for $\bar{\delta}_{r}=\widehat{\delta}_{r}=2.5 \delta_{r}^{0} ;$ - DNS filtered with $\Delta=3 \delta_{r}^{0} ; \cdot-\cdot$ F-TACLES with $\Delta=3 \delta_{r}^{0} ;---$ TFLES with $F=2.5$.
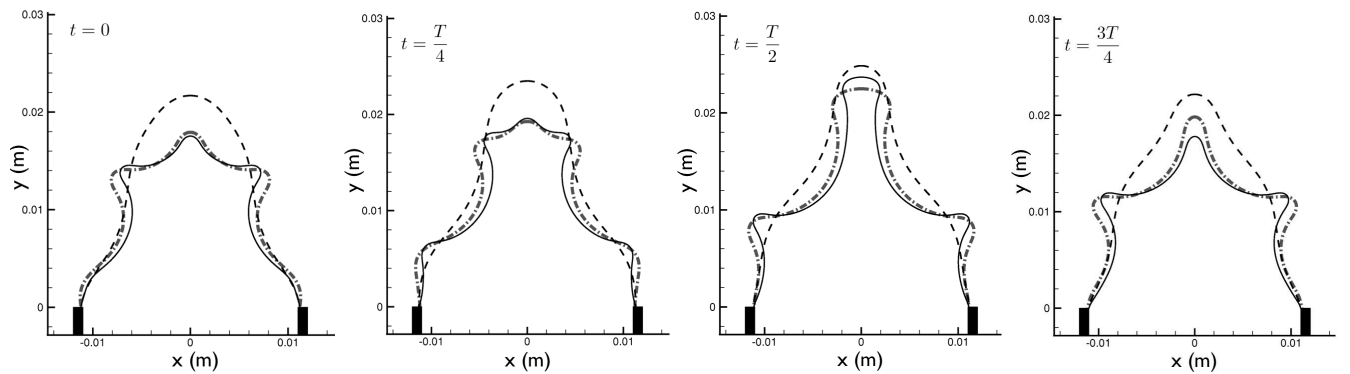

Figure 6: Iso-line $\widetilde{c}=0.5$ during a cycle for $\bar{\delta}_{r}=\widehat{\delta}_{r}=5.7 \delta_{r}^{0} ;$ - DNS filtered with $\Delta=7.9 \delta_{r}^{0} ; \cdot-\cdot$ F-TACLES with $\Delta=7.9 \delta_{r}^{0} ;---$ TFLES with $F=5.7$. 
L 


\section{List of Figures}

1 Predicted flame speed ratii as a function of $\bar{\delta}_{r} / \Delta_{x}$ for $\bar{\delta}_{r}=\widehat{\delta}_{r}=2.5 \delta_{r}^{0}$ (top) and $\bar{\delta}_{r}=\widehat{\delta}_{r}=5.7 \delta_{r}^{0}$ (bottom). $\bigcirc$ : FTACLES $S_{\Delta} / S_{l}^{0}, \mathbf{\Delta}$ : TFLES $S_{F} / S_{l}^{0}$. .

2 Relation between $\Delta / \delta_{r}^{0}$ and $F$ when $\bar{\delta}_{r}=$ $\widehat{\delta}_{r}$. Symbols: Computations. Solid line: Analytical model (Appendix A). . . .

4 Evolution of the resolved thermal thickness as a function of the thickening factor $F, \boldsymbol{\square}: \bar{\delta}_{T}, \bullet: \widehat{\delta}_{T} \ldots \ldots \ldots \ldots$

7 Heat release rate integrated over the computational domain as a function of time during a cycle. $\bar{\delta}_{r}=\widehat{\delta}_{r}=2.5 \delta_{r}^{0} ;-$ DNS; $\cdot-\cdot$ F-TACLES; --- TFLES.

3 Effect of filtering and thickening operators on the flame structure. Solid lines: F-TACLES. Dashed lines: TFLES. Without symbol: $\bar{\delta}_{r}=\widehat{\delta}_{r}=2.5 \delta_{r}^{0}$. Symbols: $\bar{\delta}_{r}=\widehat{\delta}_{r}=5.7 \delta_{r}^{0}$. a) Progress variable source term, b) Temperature, c) Ve-

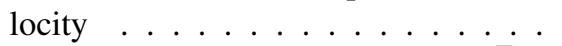
Iso-line $\widetilde{c}=0.5$ during a cycle for $\bar{\delta}_{r}=$ $\widehat{\delta}_{r}=2.5 \delta_{r}^{0} ;$ - DNS filtered with $\Delta=$ $3 \delta_{r}^{0} ; \cdot-\cdot$ F-TACLES with $\Delta=3 \delta_{r}^{0}$;

TFLES with $F=2.5$. . . . . . . . . 10

6 Iso-line $\widetilde{c}=0.5$ during a cycle for $\bar{\delta}_{r}=$ $\widehat{\delta}_{r}=5.7 \delta_{r}^{0} ;$ - DNS filtered with $\Delta=$ $7.9 \delta_{r}^{0} ; \cdot-\cdot$ F-TACLES with $\Delta=7.9 \delta_{r}^{0}$; --- TFLES with $F=5.7$. . . . . .

Heat release rate integrated over the computational domain as a function of time during a cycle. $\bar{\delta}_{r}=\widehat{\delta}_{r}=5.7 \delta_{r}^{0}$; DNS; $\cdot-\cdot$ F-TACLES; --- TFLES. 